INPLASY

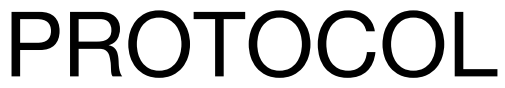

To cite: Mai et al. A scoping review protocol to systematically review published literature for managing cancer pain patients via telemedicine. Inplasy protocol 202110010. doi: 10.37766/inplasy2021.1.0010

Received: 03 January 2021

Published: 04 January 2021

Corresponding author: Yu-Fang Hao

haoyufang0903@sina.com

Author Affiliation: Center for Evidence-Based Nursing, Beijing University of Chinese Medicine

Support: School project support.

Review Stage at time of this submission: Preliminary searches.

Conflicts of interest: None.

\section{A scoping review protocol to systematically review published literature for managing cancer pain patients via telemedicine}

\author{
Mai, QL1; Zhou, YJ2; Li, XJ3; Yang, D4; Hao, YF5.
}

Review question / Objective: 1. How and by which providers, regions and countries have telemedicine of managing cancer pain been conducted so far? 2. What are the characteristics of the population included in the study? 3 . What kinds of the telemedicine tools are adopted and what are their characteristics (including availability and effectiveness)? 4. How was these studies designed and conducted? 5. What are the outcome indicators for telemedicine of cancer pain and how are they evaluated? 6. What strategies were used in the studies to optimize the feasibility, security, and compliance of telemedicine?

INPLASY registration number: This protocol was registered with the International Platform of Registered Systematic Review and Meta-Analysis Protocols (INPLASY) on 4 January 2021 and was last updated on 4 January 2021 (registration number INPLASY202110010).

\section{INTRODUCTION}

Review question / Objective: 1. How and by which providers, regions and countries have telemedicine of managing cancer pain been conducted so far? 2. What are the characteristics of the population included in the study? 3 . What kinds of the telemedicine tools are adopted and what are their characteristics (including availability and effectiveness)? 4. How was these studies designed and conducted? 5. What are the outcome indicators for telemedicine of cancer pain and how are they evaluated? 6. What strategies were 
used in the studies to optimize the feasibility, security, and compliance of telemedicine?

Condition being studied: Telemedicine, defined as Delivery of health services via remote telecommunications. This includes interactive consultative and diagnostic services. cancer was included in the category of chronic diseases by WHO, the incidence rate of cancer is on the rise year by year.Cancer pain is defined as a subjective feeling or emotional discomfort associated with or described by actual or potential tissue damage.Pain is one of the most common symptoms of cancer patients, and a survey by the International Pain Society shows that $90 \%$ of cancer patients have experienced pain at some time during their illness. The incidence of pain in patients with newly diagnosed cancer is about $25 \%$, while the incidence of pain in patients with advanced cancer is as high as $60 \%$ to $80 \%$, of which one-third are in severe pain. Up to $75 \%$ of patients with cancer will experience pain severe enough to require opioid treatment.If cancer pain is not timely and effectively controlled, patients often feel extreme discomfort, which may cause or exacerbate symptoms such as anxiety, depression, fatigue, insomnia, and loss of appetite, significantly affecting the patient's daily activities, ability to take care of themselves, social interactions, and overall quality of life.

\section{METHODS}

Search strategy: To identify original studies suitable for answering the research questions, we searched CNKI, VIP, WANFANG, CBM, PubMed, CINAHL, Cochrane Library, PschINFO and EMBASE databases since the establishment of the database. The search syntax was composed of "tele-health"'cancer pain" and their synonyms. The search was limited to studies in Chinese and English because of the costs and time involved in translating material in foreign languages such as French, German, Polish, Spanish, and Russian. Search terms include 'Neoplasms' and 'Pain' and 'telemedicine'.
Participant or population: We included Patients with diagnosed cancer and symptoms of pain and primary health care provider or specialist.

Intervention: 1. telemedicine of pain symptojms in cancer patients2.Remote means include phone, email, social network, etc.3.Include at least one telemedicine mode, such as remote monitoring, mobile applications, structured telephone management of nurses, patient self-management website, etc. Provide patient management through mobile devices in health care facilities or experts guide primary health care personnel remotely to manage patients were excluded.

Comparator: Types of control interventions included in our studies were no treatment, placebo treatment.

Study designs to be included: Published primary research, including quantitative, qualitative, mixed research are eligible for inclusion. Expert opinions or patents and not published in Chinese or English were excluded.

Eligibility criteria: Published primary research, including quantitative, qualitative, mixed research are eligible for inclusion. Expert opinions or patents and not published in Chinese or English were excluded. Participants: We included Patients with diagnosed cancer and symptoms of pain and primary health care provider or specialist. Types of interventions: 1 . telemedicine of pain symptojms in cancer patients2.Remote means include phone, email, social network, etc.3.Include at least one telemedicine mode, such as remote monitoring, mobile applications, structured telephone management of nurses, patient self-management website, etc. Provide patient management through mobile devices in health care facilities or experts guide primary health care personnel remotely to manage patients were excluded. Types of control interventions included in our studies were no treatment, placebo treatment. 
Information sources: To identify original studies suitable for answering the research questions, we searched CNKI, VIP, WANFANG, CBM, PubMed, CINAHL, Cochrane Library, PschINFO and EMBASE databases since the establishment of the database. The search syntax was composed of "tele-health" "cancer pain" and their synonyms. The search was limited to studies in Chinese and English because of the costs and time involved in translating material in foreign languages such as French, German, Polish, Spanish, and Russian.

Main outcome(s): PRISMA-ScR was used as a reference for our scoping review. Quantitative data and critical evaluation results of individual studies will be presented in tabular form. Qualitative data will be analyzed using subject matter, and the results will be briefly organized into a tabular format, with further explanations where further interpretation is required to explain the research results in depth. The data extracted from the study is presented visually or graphically so that it can be summarized or conceptualized as needed. We will elaborate on the findings that are most relevant to the research issues in this review, and describe the knowledge gaps in the research evidence and their implications.Through the critical evaluation of individual studies, our main findings will provide reference for future research and the practice of telemedicine of patients with cancer pain. The breadth of research evidence contained in this study allows us to provide a general overview of research on telemedicine of cancer pain, but it should be caution in interpreting the findings of individual patient groups.

Quality assessment / Risk of bias analysis: In this study, the report quality evaluation and bias evaluation were not carried out for the included studies, because the scoping review did not involve this process. However, we included the general characteristics of the studies, the types of studies (randomized controlled and observational studies), the data of the included statistics, and the sources of qualitative studies.
Strategy of data synthesis: PRISMA-ScR was used as a reference for our scoping review. Quantitative data and critical evaluation results of individual studies will be presented in tabular form. Qualitative data will be analyzed using subject matter, and the results will be briefly organized into a tabular format, with further explanations where further interpretation is required to explain the research results in depth. The data extracted from the study is presented visually or graphically so that it can be summarized or conceptualized as needed.

Subgroup analysis: We will conduct subgroup analysis based on the types of telemedicine (telephone, website, text word, wechat etc.) and age group, depending on data.

Sensibility analysis: In this study, the sensibility analysis were not carried out for the included studies.

Country(ies) involved: China.

Keywords: telemedicine, pain management, Cancer pain, Scoping review, Protocol.

Contributions of each author:

Author 1 - Qiu-lu Mai.

Author 2 - Ya-jing Zhou.

Author 3 - Xue-jing Li.

Author 4 - Dan Yang.

Author 5 - Yu-fang Hao. 